\title{
A rare case of candida tropicalis causing native aortic valve endocarditis in an immunocompetent host
}

\begin{abstract}
Infective Endocarditis remains a difficult to treat and life threatening condition despite advances in antimicrobial therapy and surgical intervention. ${ }^{1}$ Infective endocarditis when caused by fungal infection is an entity that holds significantly increased morbidity and mortality. We discuss a case of fungal endocarditis and its successful management.
\end{abstract}

Volume 9 Issue I - 2017

\author{
Shanmugam Balaji, Khalif Adnan, Butcher \\ Brad W, Yassin Mohamed, John R Ward DO \\ UPMC Mercy, USA
}

\begin{abstract}
Correspondence: John R Ward DO,Vice Chair of Internal Medicine, Treasurer of Medical Executive Committee, Osteopathic Program Director, Clinical Professor of Medicine, UPMC Mercy, USA, Email wardJRI@UPMC.EDU
\end{abstract}

Received: May 22, 2017 | Published: June 02, 2017

\section{Introduction}

In the context of the worsening epidemic of heroin abuse, the incidence of hospitalization for endocarditis in the United States has been increasing at a rate of $2.4 \%{ }^{2}$ annually. Despite this, fungal endocarditis remains relatively rare, accounting for only $2 \%$ of all endocarditis cases, most often occurring in immunocompromised patients or those with prosthetic valves. Here, we present a rare case of Candida tropicalis causing native aortic valve endocarditis in an immunocompetent patient.

\section{Case presentation}

A 47-year-old immunocompetent male with a medical history significant for active intravenous heroin abuse presented to the hospital with fever and respiratory distress. On admission, he was diaphoretic, febrile, and hypoxemic with an oxygen saturation of $70 \%$ on ambient air, necessitating intubation and mechanical ventilation. Chest radiography revealed a right lower lobe infiltrate concerning for pneumonia, blood cultures were obtained, and vancomycin and piperacillin-tazobactam were empirically administered. Within 48hours, blood cultures grew Candida tropicalis, and antifungal therapy with micafungin was initiated. Computed tomographic scan of the chest revealed bilateral upper lobe infiltrates and dense consolidations with air bronchograms in the bilateral posterior lobes. Transthoracic echocardiography demonstrated severe aortic thickening and aortic regurgitation but no clear evidence of vegetation despite clinical suspicion for endocarditis. Sites of metastatic infection, including endophthalmitis and septic emboli to the brain were ruled out, and antifungal therapy was adjusted to amphotericin $\mathrm{B}$ and flucytosine. A subsequent transesophageal echocardiogram revealed a $1.3 \mathrm{~cm}$ aortic valve vegetation associated with severe aortic regurgitation, for which he underwent aortic valve replacement with a bioprosthetic pericardial valve. Intra-operative cultures from the aortic valve confirmed Candida Tropicalis. He was discharged to a skilled nursing facility to complete 6 weeks of antifungal therapy.

\section{Discussion}

In the absence of appropriate treatment, which includes appropriate antifungal therapy and surgical valve replacement, fungal endocarditis has a mortality rate of $72 \% .^{3}$ Given such marked mortality and recurrence rates, a high index of suspicion is required in patients presenting with risk factors such as intravenous drug abuse, even in those without prosthetic valves or immunocompromised state. Cardiologists in regions of endemic intravenous drug abuse should have heightened clinical suspicion for less common etiologies of endocarditis. Empiric broad-spectrum antibiotic therapies, blood cultures using techniques to isolate fungal organisms, and early consultation of infectious diseases and cardiothoracic surgery should be considered in an effort to improve patient outcomes ${ }^{4-6}$ (Figure 1 \& 2).

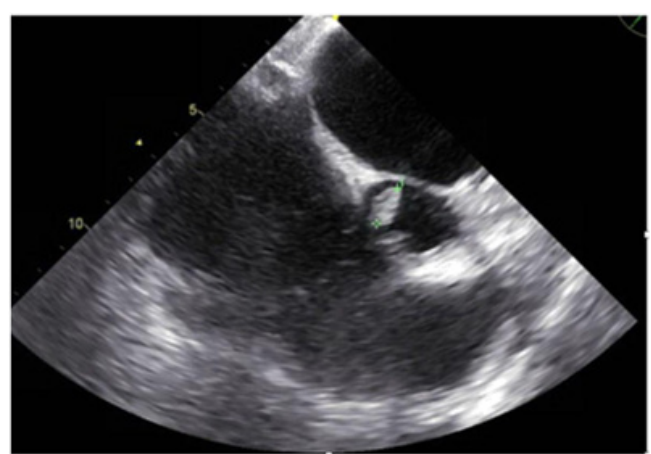

Figure I Transesophageal Echocardiogram showing a $1.3 \mathrm{~cm}$ vegetation in right coronary cusp.

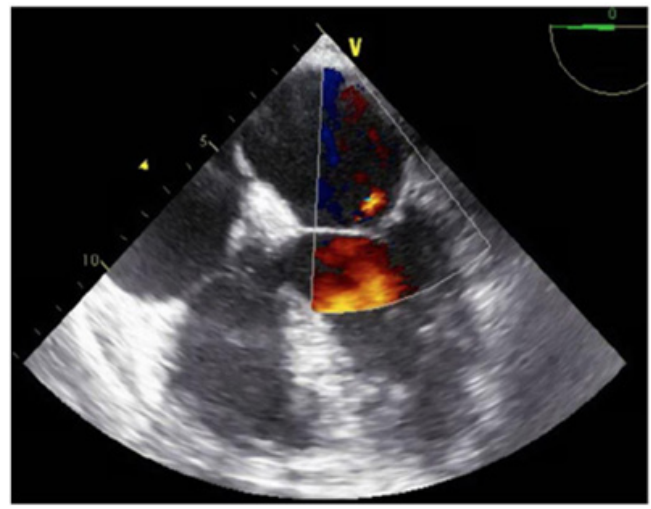

Figure 2 Transesophageal Echocardiogram showing left atrium dilatation $(4.8 \mathrm{~cm})$; and severe aortic valve regurgitation. 


\section{Acknowledgments}

None.

\section{Conflicts of interest}

Author declares there are no conflicts of interest.

\section{Funding}

None.

\section{References}

1. Nishimura. AHA/ACC Guideline for the Management of Patients with Valvular Heart Disease A Report of the American College of Cardiology/ American Heart Association. JACC. 2014;63(22):e57-185.

2. Larry MB, Walter RW, Arnold SB, et al. Infective Endocarditis in Adults: Diagnosis, Antimicrobial Therapy, and Management of Complications: A Scientific Statement for Healthcare Professionals from the American Heart Association. Circulation. 2015;132:1435.
3. Boh DH, Woolhandler S, Nardin R, et al. Infective endocarditis in the U.S., 1998-2009: a nationwide study. PLoS One. 2013;8(3):e60033.

4. Steinbach WJ, Perfect JR, Cabell $\mathrm{CH}$, et al. A meta-analysis of medical versus surgical therapy for Candida endocarditis. $J$ Infect . 2005;51(3):230-247.

5. Ellis ME, Al-Abdely $\mathrm{H}$, Sandridge A, et al. Fungal endocarditis: evidence in the world literature. Clin Infect Dis. 2001;32(1):50-62.

6. Habib G, Lancellotti P, Antunes MJ, et al. 2015 ESC Guidelines for the management of infective endocarditis The Task Force for the Management of Infective Endocarditis of the European Society of Cardiology (ESC)Endorsed by: European Association for CardioThoracic Surgery (EACTS), the European Association of Nuclear Medicine (EANM). Eur Heart J. 2015;36(44):3075-3128. 